\title{
Rheological Properties of Welan Gum in Aqueous Media ${ }^{\dagger}$
}

\author{
Masakuni TAKo and Masahiro KIRIAKI \\ Department of Agricultural Chemistry, College of Agriculture, \\ University of the Ryukyus, Nishihara, Okinawa 903-01, Japan \\ Received March 15, 1990
}

\begin{abstract}
The flow behavior and dynamic viscoelasticity of welan gum solutions were measured with a rheogoniometer. The welan gum showed shear-thinning behavior at a concentration of $0.1 \%$, but plastic behavior above $0.3 \%$ at $25^{\circ} \mathrm{C}$. The dynamic viscoelasticity increased with increasing concentration, and was scarcely changeable with increasing temperature even at $80^{\circ} \mathrm{C}$. Gelation did not occur even in a polysaccharide concentration of $1.0 \%$ at low temperature $\left(0^{\circ} \mathrm{C}\right)$. An increase of the dynamic modulus was not observed on the addition of $\mathrm{CaCl}_{2}(6.8 \mathrm{~mm})$. The dynamic viscoelasticity of welan gum solution was scarcely changeable in a wide range of $\mathrm{pH}$ from 2 to 12 . The dynamic modulus was also scarcely changeable on addition of urea $(4.0 \mathrm{M})$. Possible mode of intramolecular associations between the $\mathrm{OH}-4$ of the D-glucosyl residue and the adjacent hemiacetal oxygen atom of the L-rhamnosyl residue, and between the methyl group of the L-rhamnosyl residue and the adjacent hemiacetal oxygen atom of the D-glucosyl residue were proposed.
\end{abstract}

Welan gum is a commercial polysaccharide produced by Alcaligenes ATCC 31555, and is non-gel-forming, but gives a thermostable, highly viscous solution at a high temperature of $140^{\circ} \mathrm{C}^{1,2)}$ The main chain of the polysaccharide consists of tetrasaccharide repeating units, 3$)-\beta$-D-Glcp- $(1 \rightarrow 4)-\beta$-D-GlcpA- $(1 \rightarrow$ 4)- $\beta$-D-Glcp- $(1 \rightarrow 4)-\alpha-\mathrm{L}-\mathrm{Rhap}-(1$, and every $\mathrm{D}-$ glucosyl residue next to the D-glucuronosyl residue is substituted at $\mathrm{O}-3$ by either an $\alpha$-L-rhamnosyl or $\alpha$-L-mannosyl residue in the ratio of $2: 1 .^{3)}$ The welan gum has approximately one acetyl group for every two repeating units, but the position of acetylation in the chain is not known. The primary structure of welan gum is similar to that of gellan gum which also consists of the same tetrasaccharide repeating units without side chains. ${ }^{4,5)}$ The gellan gum forms a weak elastic gel, but it forms a stiff, brittle gel on deacetylation. ${ }^{6)}$

We have proposed a possible mode of intra- and inter-molecular associations of gellan gum molecules in aqueous solution. ${ }^{7)}$
The intramolecular hydrogen bonding may take place between the $\mathrm{OH}-4$ of the D-glucosyl residue and the adjacent hemiacetal oxygen atom of the L-rhamnosyl residue, and between $\mathrm{OH}-3$ of the D-glucosyl residue and the adjacent hemiacetal oxygen atom of the D-glucuronosyl residue, to make the gellan gum molecule rigid. The intermolecular association may take place between the methyl group and the hemiacetal oxygen atom of the L-rhamnosyl residues in different molecules with van der Waals interaction by a cage effect. In the presence of $\mathrm{CaCl}_{2}$, an intermolecular $\mathrm{Ca}^{2+}$ bridge, as in l-carrageenan ${ }^{8)}$ may also take place between the carboxyl oxygen atom of the D-glucuronosyl residues in different molecules with ionic bonding.

Physical properties of welan gum in aqueous solutions have been reported by Crescenzi et al. ${ }^{9)}$ and Brant et al. ${ }^{10,11)}$ Brant et al. have concluded that the welan gum molecule is involved in intramolecular van der Waals interaction and hydrogen bonding between side chain and backbone in aqueous solution.

$\uparrow$ Presented at the Annual Meeting of the Japan Society for Bioscience, Biotechnology, and Agrochemistry, Fukuoka, March 30-April 2, 1990. 
These polysaccharides, gellan and welan gum, provide an interesting opportunity to investigate polysaccharide structure-function relationships. We report herein the flow behavior and dynamic viscoelasticity of welan gum, and its rheological properties are analyzed with respect to its association characteristics, in comparison with those of deacetylated gellan gum. ${ }^{\text {7) }}$

\section{Materials and Methods}

Materials. Welan gum was kindly supplied by Kelco Co., Ltd. and was dissolved in hot water $\left(60^{\circ} \mathrm{C}\right)$ as a $0.1 \%$ solution. The solution was heated at $90^{\circ} \mathrm{C}$ for $20 \mathrm{~min}$, and then cooled to room temperature, centrifuged at $23,000 \times g$ for $1 \mathrm{hr}$, and filtered through Celite 545 (which had been treated with boiling $3 \mathrm{M} \mathrm{HCl}$ for $30 \mathrm{~min}$ and washed with distilled water until the $\mathrm{pH}$ was 6.5 ). In the presence of $0.1 \% \mathrm{KCl}$, ethanol (2 vols.) was added to the filtrate, and then the precipitate was dried in vacuo. Purified welan gum was redissolved in hot water and the solution deionized by passage through a column of Amberlite $120\left(\mathrm{H}^{+}\right)$, and then neutralized with $50 \mathrm{~mm} \mathrm{KOH}$. The solution was filtered through Celite 545 again. Ethanol (2 vols.) was added to the filtrate in the presence of $0.05 \% \mathrm{KCl}$, and the precipitate was dried in vacuo.

The gellan gum used was identical with that in our previous study; ${ }^{7)}$ it was obtained from Sanei Kagaku Co., Ltd. Gellan gum was dissolved in hot water $\left(95^{\circ} \mathrm{C}\right)$ at a concentration of $0.2 \%$ then filtered through Celite 545 keeping the temperature at $70^{\circ} \mathrm{C}, \mathrm{KCl}$ was added to a concentration of $0.05 \%$, and then ethanol ( 2 vols.) was added. The precipitate was dried in vacuo. Purified gellan gum was redissolved in hot water $\left(90^{\circ} \mathrm{C}\right)$ and the solution deionized, at $65^{\circ} \mathrm{C}$ by passage through a column of Amberlite IR-120 $\left(\mathrm{H}^{+}\right)$, and then neutralized with $50 \mathrm{~mm}$ $\mathrm{KOH}$. The solution was filtered through Celite 545 again. Ethanol ( 2 vols.) was added to the filtrate in the presence of $0.05 \% \mathrm{KCl}$, and the precipitate was dried in vacuo.

Specific rotation. Specific rotation was measured at $589 \mathrm{~nm}$ with an automatic digital polarimeter DIP 180 (Japan Sepctroscopic Co., Ltd.) for a $0.3 \%$ aqueous solution of welan gum.

Infrared spectroscopy. The identity of the polysaccharide as acetylated welan gum was checked by infrared spectroscopy. The spectra were recorded with an infrared spectrophotometer IR 440 (Shimadzu Seisakusho Co., Ltd.) for samples dispersed in $\mathrm{KBr}$ discs.

Viscosity and dynamic Viscoelasticity measurements. Viscosity at various shear rates $\left(1.1995 .03 \mathrm{sec}^{-1}\right)$ and dynamic viscoelasticity at a steady angular velocity
(3.77 rad/sec) were measured with a rheogoniometer consisting of a coaxial cylinder $(1.8 \mathrm{~cm}$ diam.) and rotating outer cylinder ( $2.2 \mathrm{~cm}$ diam.), $6.0 \mathrm{~cm}$ long (IR 103, Iwamoto Seisakusho Co., Ltd.). The temperature of the sample was controlled by circulating oil with a Thermo-cool (LCH $-130 \mathrm{~F}$, Toyo Co., Ltd.) over a temperature ranging from 0 to $85^{\circ} \mathrm{C}$ and raised at a rate of $1{ }^{\circ} \mathrm{C} / \mathrm{min}$ by steps. Shear rate $(D)$, shear stress $(S)$, and apparant viscosity $\left(\eta_{\mathrm{ap}}\right)$ were calculated with the equation of Margules. ${ }^{12}$ ) Dynamic viscosity $\left(\eta^{\prime}\right)$ and elasticity $\left(G^{\prime}\right)$ were calculated by a modification of Markovitz's equation. ${ }^{13}$ ) The loss $\operatorname{tangent}(\tan \delta)$ was calculated from the relationship tan $\delta=G^{\prime \prime} / G^{\prime}$, where $G^{\prime \prime}$ is the loss modulus $\left(\omega \eta^{\prime}\right)$ and $\omega$ is the angular velocity of the outer cylinder.

\section{Results}

The identity of the polysaccharide as an acetylated welan gum was established by infrared absorption at $1730 \mathrm{~cm}^{-1}$.

To compare the rheological behaviors of welan gum to those of gellan gum, the viscosity and dynamic viscoelasticity were measured under the same conditions as those of our previous study. ${ }^{7)}$ The flow curves, at $25^{\circ} \mathrm{C}$, of

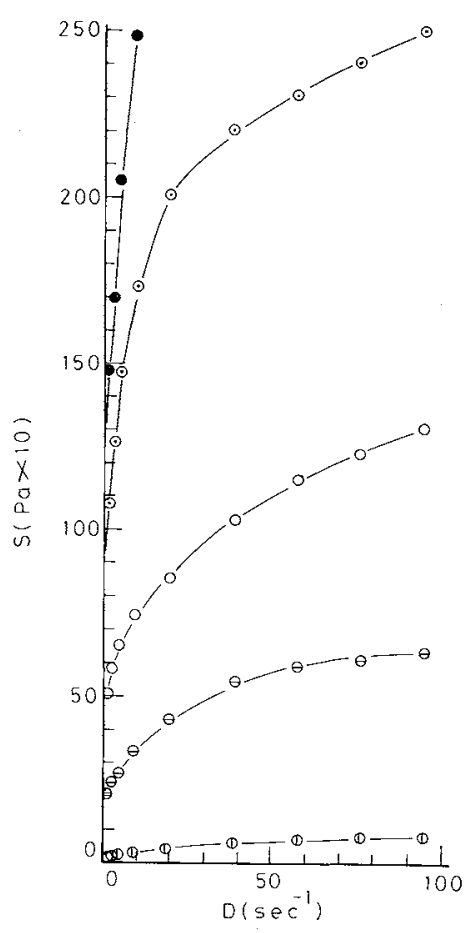

Fig. 1. Flow Curves of Welan Gum at Various Concentrations and at $25^{\circ} \mathrm{C}$.

(D) $0.1 \% ; \ominus, 0.3 \% ; \bigcirc, 0.5 \% ; \odot, 0.8 \% ; \bigcirc, 1.0 \%$. 
welan gum at various concentrations are shown in Fig. 1. The flow curves at a concentration of $0.1 \%$ approximated to shear-thinning behavior, but to plastic flow behavior above $0.3 \%$, and the yield value was estimated to be $20,50,90$, and $130 \mathrm{~Pa} \times 10$ at $0.3,0.5,0.8$, and $1.00 \%$, respectively. The flow curves of welan gum solutions shifted over high shear-stress in proportion to the concentrations. This tendency differed from that of gellan gum solutions, where the flow curves shifted to a very low shear-stress below $0.9 \%$. This might be caused by the breakdown of an intermolecular association of gellan gum molecules, since at $1.0 \%$ solution it shifted over to very high shear-stress and showed plastic behavior, the yield value of which was estimated to be $10 \mathrm{~Pa} \times 10{ }^{7)}$

As reported previously, ${ }^{7)}$ the dynamic modulus of gellan gum solution was very large at a concentration of $0.8 \%$ at low temperature $\left(0^{\circ} \mathrm{C}\right)$, and decreased rapidly with increases in termperature. The dynamic modulus of welan gum in a $0.8 \%$ solution was very large at low temperatures, but it stayed large during

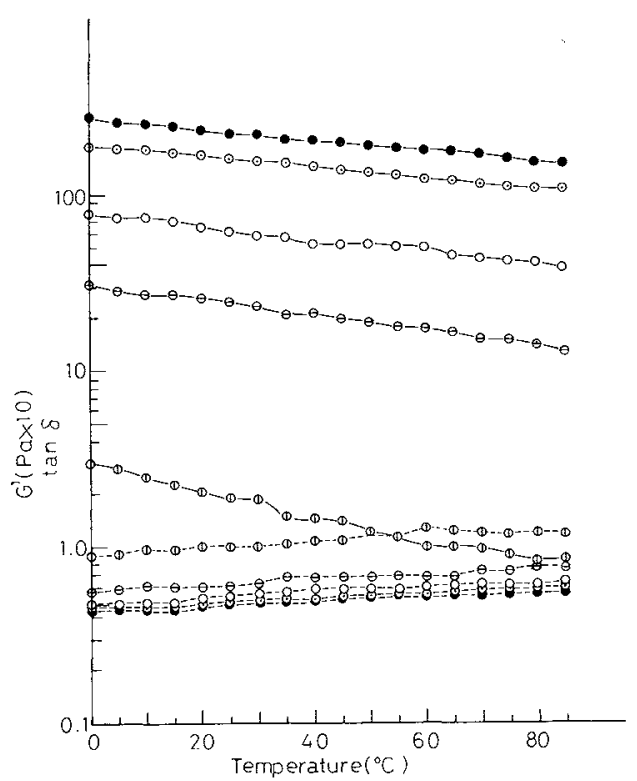

Fig. 2. Effects of Temperature on the Dynamic Modulus and $\tan \delta$ of Welan Gum at Various Concentrations.

(1) $0.1 \% ; \ominus, 0.3 \% ; \bigcirc, 0.5 \% ; \odot, 0.8 \%$; dynamic modulus; -----, $\tan \delta$. incresases in temperature even to $85^{\circ} \mathrm{C}$, as shown in Fig. 2. Despite the larger dynamic modulus than that of gellan gum, ${ }^{7)}$ gelation did not take place even in a $1.0 \%$ solution of welan gum at low temperature. On ther other hand, the $\tan \delta$ value of the welan gum decreased from 0.52 to 0.43 with increase in concentration from 0.5 to $1.0 \%$ at low temperature $\left(0^{\circ} \mathrm{C}\right)$. The values at both concentrations were lower than those of gellan gum, ${ }^{7}$ but almost agreed with those of native xanthan. ${ }^{14)}$

A very large dynamic modulus was observed on addition of $\mathrm{CaCl}_{2}(6.8 \mathrm{~mm})$ to a $0.2 \%$ solution of gellan gum, and increased with the increase of temperature up to $80^{\circ} \mathrm{C}$, then it decreased rapidly with further increases in temperature (Fig. 3). This indicates that the gellan gum molecules associate tightly with $\mathrm{Ca}^{2+}$, where carboxyl groups of D-glucuronosyl residues may contribute to cation bridges on the different molecules with ionic bonding. On the contary, the dynamic modulus of welan gum solution $(0.2 \%)$ decreased a little on addition of $\mathrm{CaCl}_{2}(6.8 \mathrm{~mm})$, indicating that

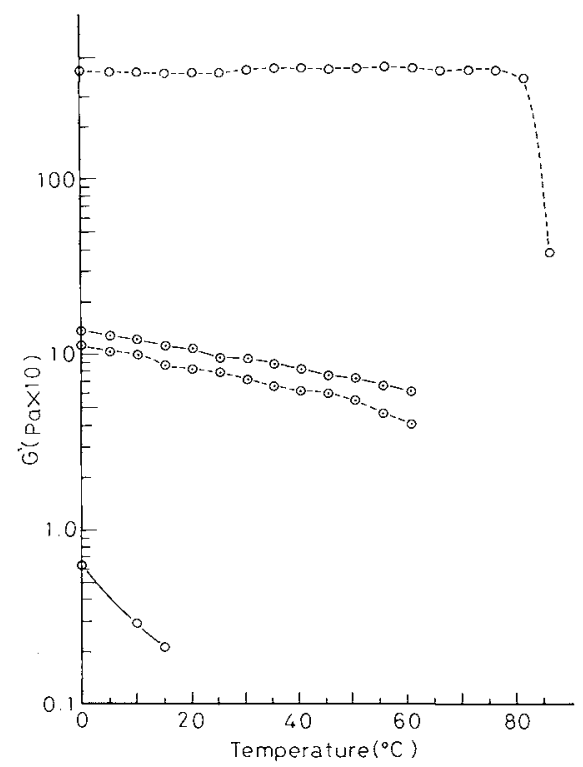

Fig. 3. Effects of Temperature on the Dynamic Modulus, at $3.77 \mathrm{rad} / \mathrm{sec}$, for $0.2 \%$ Aqueous Solution of Welan and Gellan Gums with Addition of $\mathrm{CaCl}_{2}$.

$\odot$, welan gum; $\bigcirc$, gellan gum. - , polysaccharide alone; -.---, with addition of $\mathrm{CaCl}_{2}(6.8 \mathrm{~mm})$. 
Table I. Specific Rotation of Welan Gum Solution at $589 \mathrm{~nm}^{a}$

\begin{tabular}{cccccccccc}
\hline & \multicolumn{1}{c}{ Temperature $\left({ }^{\circ} \mathrm{C}\right)$} \\
\cline { 2 - 9 } & 10 & 20 & 25 & 30 & 40 & 50 & 60 & 70 \\
\hline Welan gum & -45 & -50 & -53 & -53 & -53 & -53 & -56 & -60 \\
\hline
\end{tabular}

a For a solution of $0.3 \%$ in water.

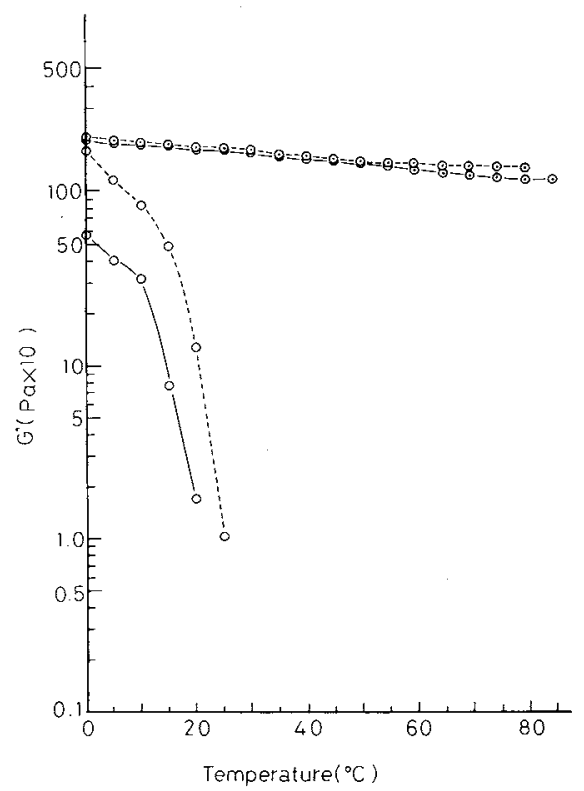

Fig. 4. Effects of Temperature on the Dynamic Modulus, at $3.77 \mathrm{rad} / \mathrm{sec}$, for $0.8 \%$ Aqueous Solution of Welan and Gellan Gums with Addition of Urea.

$\odot$, welan gum; $\bigcirc$, gellan gum. $\_$, polysaccharide alone; -.--, with addition of urea $(4.0 \mathrm{M})$

the side-chain, substituted at $0-3$ of every D-glucosyl residue next to the D-glucuronosyl residue by either an $\alpha$-L-rhamnosyl or $\alpha$-Lmannosyl rasidue in the ratio of $2: 1$, of welan gum molecules prevents the formation of $\mathrm{Ca}^{2+}$ bridges on different molecules.

Though the dynamic modulus of gellan gum $(0.8 \%)$ was higher than that of the polymer alone upon the addition of urea $(4.0 \mathrm{M})$ and gel formation was observed at low temperature $\left(0^{\circ} \mathrm{C}\right)$, for welan gum, it was nearly independent on addition of urea and stayed very large during increases in temperature as in the polysaccharide alone (Fig. 4). Despite having a larger dynamic modulus in the welan gum

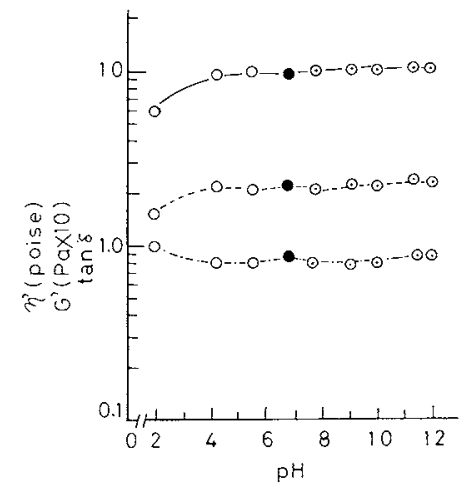

Fig. 5. Effects of $\mathrm{pH}$ on the Dynamic Viscoelasticity at $3.77 \mathrm{rad} / \mathrm{sec}$, for Aqueous Solution of $0.2 \%$ Welan Gum at $25^{\circ} \mathrm{C}$.

The $\mathrm{pH}$ value was adjusted with $100 \mathrm{~mm} \mathrm{HCl}(\mathrm{O})$ or $\mathrm{KOH}$ $(\odot) ; \odot$, welan gum alone. ---.-, dynamic viscosity; - , dynamic modulus; -...-., $\tan \delta$.

solution $(0.8 \%)$ on addition of urea than that of gellan gum, gel formation was not observed, suggesting that the side chain of welan gum molecules prevents an intermolecular association.

The dynamic viscoelasticity of the welan gum solution $(0.2 \%)$ was nearly independent of $\mathrm{pH}$ change between 2 and 12 upon the addition of $100 \mathrm{~mm} \mathrm{HCl}$ or $\mathrm{KOH}$, as shown in Fig. 5. This tendency was inconsistent with that of gellan gum $^{7)}$, in which a very large dynamic modulus was observed in acid and alkaline range after reaching $\mathrm{pH} 2.6$ and 11.2 , by addition of $100 \mathrm{~mm}$ $\mathrm{HCl}$ or $\mathrm{Ca}(\mathrm{OH})_{2}$.

The specific rotation, at $0.3 \%$, of welan gum at various temperatures is summarized in Table I. The specific rotation decreased a little with increasing temperature up to $25^{\circ} \mathrm{C}$, where it stayed constant at $[\alpha]_{D}-53^{\circ}$; after the temperature reached $50^{\circ} \mathrm{C}$, the specific rotation decreased a little with further increases of 
temperature. These data indicated that the welan gum molecules may adopt an ordered conformation in aquous solution. ${ }^{7,15-17)}$

\section{Discussion}

Though the primary structure of welan gum is similar to that of gellan gum, the rheological characteristics of the former differ from those of the latter. ${ }^{7)}$ The welan gum showed a plastic behavior even in a solution of low concentration $(0.3 \%)$, and the yield value increased with increasing concentration at room temperature $\left(25^{\circ} \mathrm{C}\right)$. Gelation did not occur even in a solution of high concentration $(1.0 \%)$ at low temperature $\left(0^{\circ} \mathrm{C}\right)$ and with addition of $\mathrm{CaCl}_{2}$ $(6.8 \mathrm{~mm})$. The $\tan \delta$ value of the welan gum decreased from 0.52 to 0.43 with increasing concentration from 0.5 to $1.0 \%$, as in xanthan. ${ }^{14)}$ The dynamic viscoelasticity was scarcely changed with increasing temperature even at $85^{\circ} \mathrm{C}$. The specific rotation decreased a little with increasing temperature up to $25^{\circ} \mathrm{C}$, where it kept constant, after the temperature reached $50^{\circ} \mathrm{C}$, the specific rotation decreased a little again with a further increase of the temperature. These results suggest that the welan gum molecules are involved in intramolecular associations, as are xanthan molecules. ${ }^{15)}$

Intramolecular hydrogen bonding seems to be built on the $\alpha$-L- $(1 \rightarrow 3)$-linkage in gellan ${ }^{7)}$ and agarose ${ }^{17)}$ molecules, as proposed in previous studies. To interpret the results, we propose that the welan gum molecule is involved in an intramolecular association, as illustrated in Scheme 1, between the $\mathrm{OH}-4$ of the D-glucosyl residue and the adjacent

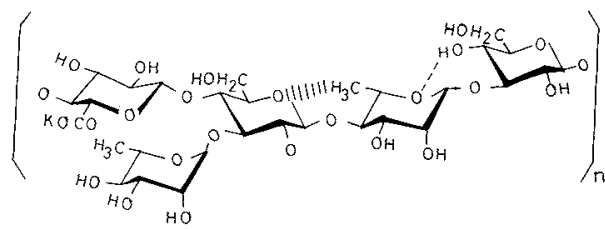

Scheme 1. Possible Mode of Intramolecular Associations in Welan Gum Molecule in Aqueous Solution.

The dotted line refers to hydrogen bonding and the broken line to van der Waals interaction. hemiacetal oxygen atom of the L-rhamnosyl residue with a hydrogen bond. This bond is likely owing to the flexibility of the $\alpha-\mathrm{L}-(1 \rightarrow 3)$ linkage ${ }^{17)}$ between L-rhamnosyl and D-glucosyl residues as in the gellan gum molecule ${ }^{7)}$ on which the L-rhamnosyl residue may adopt a ${ }^{4} \mathrm{C}_{1}$ pyranose-ring conformation. ${ }^{18)}$

The dynamic viscoelasticity of welan gum was scarcely changed by increasing temperature, suggesting that the methyl groups of the L-rhamnosyl residues may contribute to the other intramolecular association as in the xanthan molecule ${ }^{15}$ in which methyl groups of the acetyl and pyruvate residues may contribute to an intra- and inter-molecular association at high temperatures. Thus we also propose a mode of intramolecular association between the methyl group of the L-rhamnosyl residue and the adjacent hemiacetal oxygen atom of the D-glucosyl residue with van der Waals interaction ${ }^{10)}$ (Scheme 1). Though the L-rhamnosyl residue may adopt a ${ }^{4} \mathrm{C}_{1}$ pyranose-ring conformation in the gellan gum molecule on which change in conformation may take place by heating from ${ }^{1} \mathrm{C}_{4}$ to ${ }^{4} \mathrm{C}_{1}$ pyranose-ring, in the welan gum molecule, it may exist in a ${ }^{1} \mathrm{C}_{4}$ pyranose-ring conformation. ${ }^{7)}$ Such a conformational change of L-rhamnosyl residue may contribute to the gel formation for gellan gum, in which the methyl group of the L-rhamnosyl residue may take part in the intermolecular van der Waals interaction, as reported previously. ${ }^{7)}$ As suggested by Brant et al., ${ }^{10)}$ the side chain, either Lrhamnosyl or L-mannosyl rasidue in the ratio of $2: 1$, of welan gum molecules may also take part in an intramolecular van der Waals interaction or hydrogen bonding with the D-glucuronosyl residue of the backbone.

Although a very large dynamic modulus was observed on addition of $\mathrm{CaCl}_{2}(6.9 \mathrm{~mm})$ to a $0.2 \%$ solution of gellan gum at low temperature, a little decrease in the dynamic modules was observed for welan gum solution. This indicates that the side chains of welan gum prevent the formation of $\mathrm{Ca}^{2+}$ bridges between the carboxyl groups of the D-glucuronosyl residues on the different molecules. The gelation 
up to a high temperature $\left(80^{\circ} \mathrm{C}\right)$ for gellan gum solution in the presence of $\mathrm{CaCl}_{2}$ and or urea suggests that a hydrophobic interac$\operatorname{tion}^{19-21)}$ between the methyl groups of the L-rhamnosyl residues on different molecules may participate to the interaction in addition to the intra- and inter-molecular hydrogen bond and van der Waals interaction. ${ }^{7)}$

Accordingly, the model (Scheme 1) provided an explanation not only for the rheological characteristics of the welan gum but also of those of the gellan gum in aqueous media. ${ }^{7}$ The welan gum molecule may keep an ordered, rigid, less extended, and 2-fold helical conformation in aquous solution, as in the solid state. $^{22)}$

\section{References}

1) K. S. Kang, G. T. Veeder and I. W. Cottrell, Prog. Ind. Microbiol., 19, 231 (1983).

2) P. A. Sandford, I. W. Cottrell and D. J. Pattit, Pure \& Appl. Chem., 56, 879 (1984).

3) P.-E. Jansson, B. Lindberg, G. Wildmalm and P. A. Sandford, Carbohydr. Res., 139, 217 (1985).

4) M. A. O'Neil, R. R. Selvender and V. J. Morris, Carbohydr. Res., 124, 123 (1983).

5) P.-E. Jansson, B. Lindberg and P. A. Sandford, Carbohydr. Res., 124, 135 (1983).

6) V. Carroll, G. R. Chilvers, D. Franklin, M. J. Miles,
V. J. Morris and S. G. Ring, Carbohydr. Res., 114 181 (1983).

7) M. Tako, A. Sakae and S. Nakamura, Agric. Biol. Chem., 53, 771 (1989).

8) M. Tako, S. Nakamura and Y. Kohda, Carbohydr. Res., 161, 247 (1987)

9) V. Crescenzi, M. Dentini and I. C. M. Dea, Carbohydr. Res., 160, 283 (1987).

10) T. A. Talashek and D. A. Brant, Carbohydr. Res., 160, 303 (1987).

11) R. Urbani and D. A. Brant, Carbohydr. Polym., 11, 169 (1989).

12) J. Harris, "Rheology and Non-Newtonian Flow," Longman, New York, 1977, p. 28.

13) H. Markovitz, J. Appl. Phys., 23, 1070 (1952).

14) M. Tako, T. Nagahama and D. Nomura, Nippon Nōgeikagaku Kaishi, 51, 513 (1977).

15) M. Tako and S. Nakamura, Agric. Biol. Chem., 53, 1941 (1989).

16) A. Hayashi, K. Kinoshita and M. Kuwano, Poly. J., 9, 219 (1977).

17) M. Tako and S. Nakamura, Carbohydr. Res., 180, 277 (1988).

18) R. E. Reeves, $A d v$. in Carbohydr. Chem., 6, 107 (1951).

19) G. Nemethy and H. A. Scheraga, J. Phys. Chem., 66, 1773 (1962).

20) P. Dubin and U. P. Strauss, J. Phys. Chem., 77, 1427 (1973).

21) E. R. Morris and I. T. Norton, "Aggregation Process in Solution," ed. by E. Wyn-Jones and J. Gormally, Elsevier, Amsterdam, 1983, p. 549.

22) P. T. Attwool, E. D. T. Atkins, M. J. Miles and V. J. Morris, Carbohydr. Res., 148, C1 (1986). 